\title{
Investigation on the Catalytic Behavior of
}

\author{
Alkali Metals and Alkaline Earth Metals on
} the Biomass Pyrolysis Assisted with Real-time

\section{Monitoring}

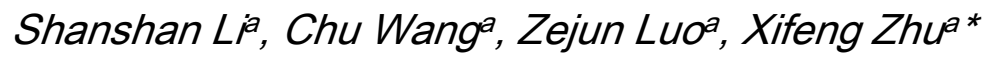

a Department of Thermal Science and Energy Engineering, University of Science and Technology of China,96 Jinzhai Road, Hefei, Anhui 230026, P. R. China

*Corresponding author: Tel: +86 551 63600040; E-mail address: xfzhu@ustc.edu.cn

Figure S1 Time-resolved mass spectra of RH recorded at 8s, 16s, 24s, and 32s

Figure S2 The accumulated intensity of the typical markers within 80 s

Table S1 The identification of the compounds in the pyrolysis volatiles at $550{ }^{\circ} \mathrm{C}$ 

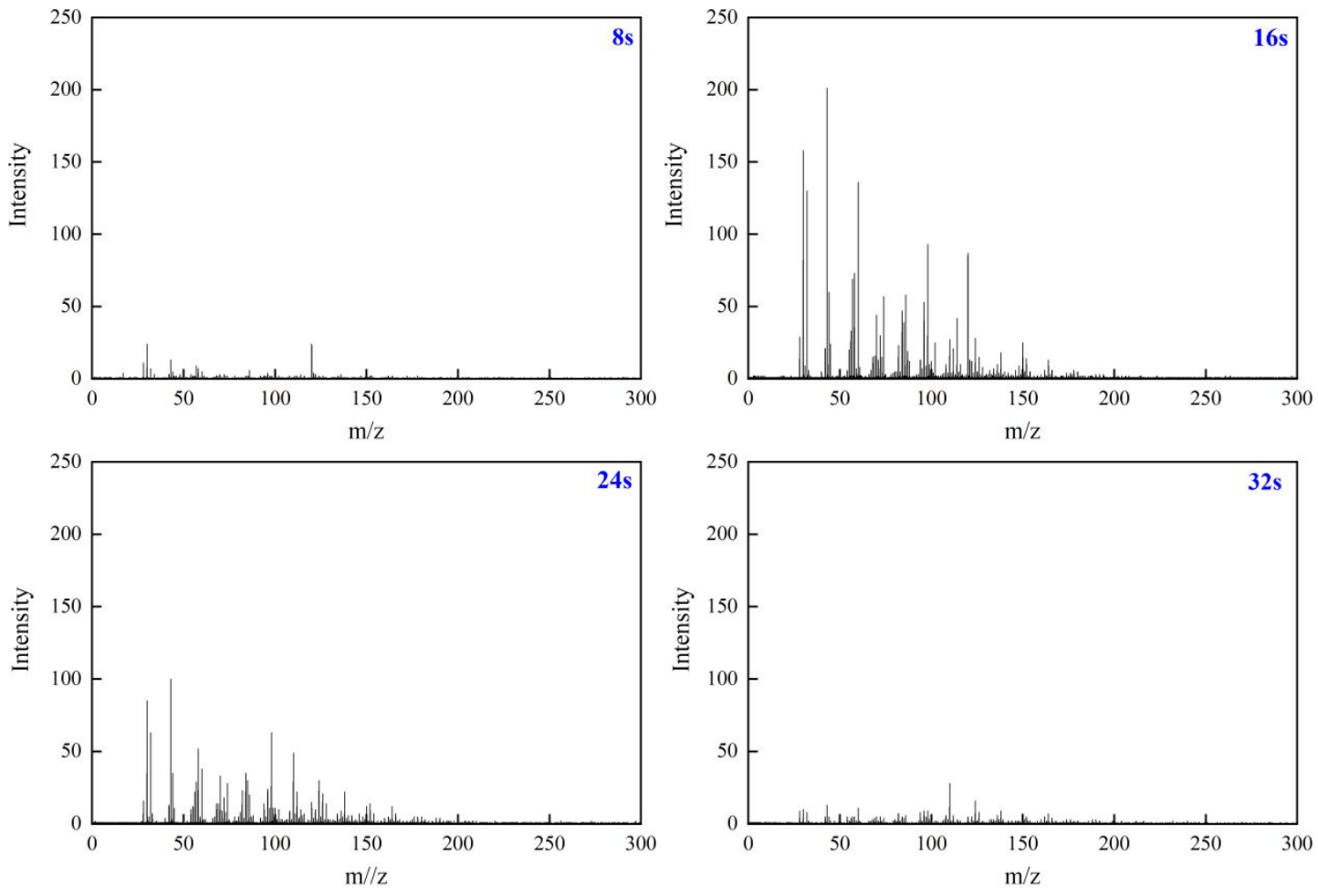

Figure S1 Time-resolved mass spectra of RH recorded at $8 \mathrm{~s}, 16 \mathrm{~s}, 24 \mathrm{~s}$, and $32 \mathrm{~s}$ 


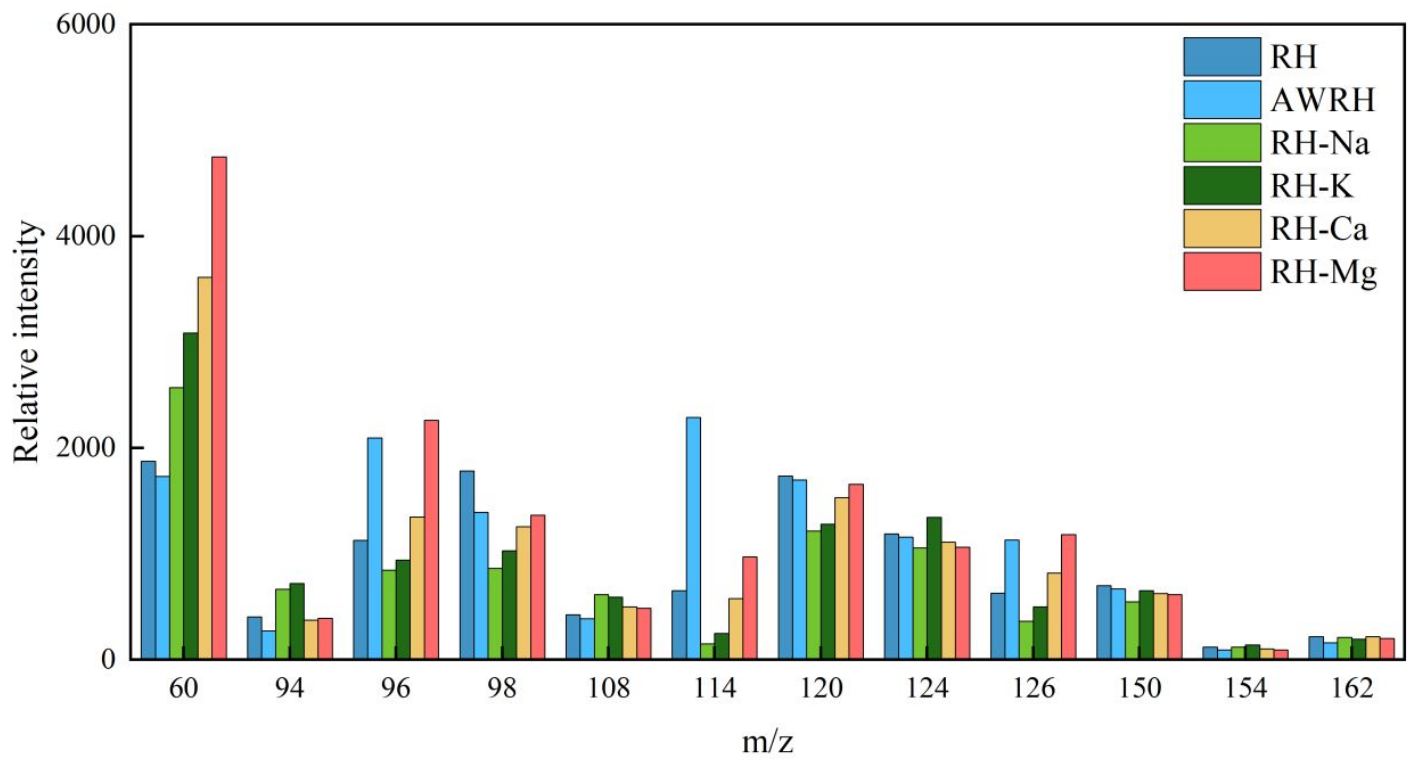

Figure S2 The accumulated intensity of the typical markers within 80 s 
Table S1 The identification of the compounds in the pyrolysis volatiles at $550{ }^{\circ} \mathrm{C}$

\begin{tabular}{|c|c|c|c|c|c|c|c|c|c|}
\hline \multirow{2}{*}{$\begin{array}{l}\text { Peak time } \\
(\min )\end{array}$} & \multirow{2}{*}{ Compounds } & \multirow{2}{*}{$\mathrm{m} / \mathrm{z}$} & \multirow{2}{*}{ Formula } & \multicolumn{6}{|c|}{ Relative peak area (\%) } \\
\hline & & & & RH & AWRH & RH-Na & RH-K & $\mathrm{RH}-\mathrm{Ca}$ & RH-Mg \\
\hline & Acids & & & 5.19 & 2.83 & 8.96 & 9.80 & 9.41 & 11.05 \\
\hline 2.30 & Acetic acid & 60 & $\mathrm{C} 2 \mathrm{H} 4 \mathrm{O} 2$ & 4.26 & 2.83 & 8.38 & 9.21 & 8.58 & 10.27 \\
\hline \multirow[t]{2}{*}{3.49} & Propanoic acid & 74 & $\mathrm{C} 3 \mathrm{H} 6 \mathrm{O} 2$ & 0.93 & -- & 0.58 & 0.59 & 0.83 & 0.78 \\
\hline & Aldehydes & & & 5.11 & 2.61 & 4.12 & 3.29 & 1.92 & 2.08 \\
\hline 1.88 & Glycolaldehyde & 60 & $\mathrm{C} 2 \mathrm{H} 4 \mathrm{O} 2$ & 1.06 & 1.09 & 0.58 & -- & 0.67 & 0.72 \\
\hline 2.02 & 3-Methoxy-propionaldehyde & 88 & $\mathrm{C} 4 \mathrm{H} 8 \mathrm{O} 2$ & 2.22 & 1.52 & 1.96 & 1.79 & 1.25 & 1.36 \\
\hline \multirow[t]{2}{*}{4.97} & Succinaldehyde & 86 & $\mathrm{C} 4 \mathrm{H} 6 \mathrm{O} 2$ & 1.84 & -- & 1.59 & 1.50 & -- & -- \\
\hline & Linear ketones & & & 0.76 & -- & 1.44 & 1.20 & 0.74 & -- \\
\hline \multirow[t]{2}{*}{2.57} & Hydroxypropanone & 74 & $\mathrm{C} 3 \mathrm{H} 6 \mathrm{O} 2$ & 0.76 & -- & 1.44 & 1.20 & 0.74 & -- \\
\hline & Cyclopentanediones & & & 3.10 & 0.86 & 2.86 & 3.11 & 2.77 & 1.91 \\
\hline 10.82 & 1,2-Cyclopentanedione & 98 & $\mathrm{C} 5 \mathrm{H} 6 \mathrm{O} 2$ & 1.93 & 0.86 & 1.15 & 1.35 & 1.33 & 1.17 \\
\hline \multirow[t]{2}{*}{15.65} & Methyl cyclopentenolone & 112 & C6H8O2 & 1.17 & -- & 1.72 & 1.76 & 1.44 & 0.74 \\
\hline & Furans & & & 2.17 & 6.12 & 2.18 & 2.34 & 3.08 & 4.41 \\
\hline 6.74 & Furfural & 96 & $\mathrm{C} 5 \mathrm{H} 4 \mathrm{O} 2$ & 1.50 & 3.32 & 2.18 & 2.34 & 2.34 & 3.73 \\
\hline 10.26 & 2(5H)-Furanone & 84 & $\mathrm{C} 4 \mathrm{H} 4 \mathrm{O} 2$ & 0.67 & 1.31 & -- & -- & -- & 0.68 \\
\hline 18.29 & $\begin{array}{l}\text { 4-Hydroxy-2,5-dimethyl- } \\
3(2 \mathrm{H}) \text { furanone }\end{array}$ & 128 & $\mathrm{C} 6 \mathrm{H} 8 \mathrm{O} 3$ & -- & 0.47 & -- & -- & -- & -- \\
\hline 21.78 & $\begin{array}{l}\text { 2-methoxy-2 3-dihydro-3- } \\
\text { furancarboxaldehyde }\end{array}$ & 128 & $\mathrm{C} 6 \mathrm{H} 8 \mathrm{O} 3$ & -- & -- & -- & -- & 0.74 & -- \\
\hline \multirow[t]{2}{*}{25.56} & 5-Hydroxymethylfurfural & 126 & C6H6O3 & -- & 1.02 & -- & -- & -- & -- \\
\hline & Phenols & & & 37.33 & 30.83 & 43.18 & 42.61 & 30.22 & 26.10 \\
\hline 13.76 & Phenol & 94 & C6H6O & 0.65 & -- & 1.35 & 1.63 & 0.57 & 0.67 \\
\hline 17.12 & p-Cresol & 108 & $\mathrm{C} 7 \mathrm{H} 8 \mathrm{O}$ & -- & -- & 0.55 & 0.68 & -- & -- \\
\hline 18.15 & m-Cresol & 108 & $\mathrm{C} 7 \mathrm{H} 8 \mathrm{O}$ & 0.54 & 0.69 & 0.92 & 0.96 & 0.59 & 0.62 \\
\hline 18.43 & Guaiacol & 124 & $\mathrm{C} 7 \mathrm{H} 8 \mathrm{O} 2$ & 3.32 & 1.47 & 5.95 & 6.31 & 1.85 & 1.47 \\
\hline 22.44 & 4-Ethylphenol & 122 & $\mathrm{C} 8 \mathrm{H} 10 \mathrm{O}$ & -- & -- & 0.49 & 0.54 & -- & -- \\
\hline 23.34 & 2-Methoxy-4-methylphenol & 138 & $\mathrm{C} 8 \mathrm{H} 10 \mathrm{O} 2$ & 2.82 & 3.52 & 1.84 & 1.55 & 2.33 & 2.14 \\
\hline 24.38 & Hydroquinone & 110 & $\mathrm{C} 6 \mathrm{H} 6 \mathrm{O} 2$ & -- & -- & 0.73 & 0.80 & -- & -- \\
\hline 24.85 & 4-Vinylphenol & 120 & $\mathrm{C} 8 \mathrm{H} 8 \mathrm{O}$ & 9.44 & 6.68 & 7.43 & 6.85 & 7.57 & 7.79 \\
\hline 27.28 & 4-Ethyl guaiacol & 152 & $\mathrm{C} 9 \mathrm{H} 12 \mathrm{O} 2$ & 1.24 & 0.58 & 2.16 & 1.89 & 1.37 & 1.33 \\
\hline 28.84 & 4-Vinyl guaiacol & 150 & $\mathrm{C} 9 \mathrm{H} 10 \mathrm{O} 2$ & 7.74 & 7.33 & 9.33 & 9.14 & 6.38 & 5.98 \\
\hline 30.52 & 2,6-Dimethoxyphenol & 154 & $\mathrm{C} 8 \mathrm{H} 10 \mathrm{O} 3$ & 1.00 & 0.53 & 2.11 & 2.13 & 0.47 & -- \\
\hline 30.72 & 5-Allylguaiacol & 164 & $\mathrm{C} 10 \mathrm{H} 12 \mathrm{O} 2$ & 0.91 & 0.90 & 0.64 & 0.58 & 0.75 & 0.56 \\
\hline 32.51 & Vanillin & 152 & $\mathrm{C} 8 \mathrm{H} 8 \mathrm{O} 3$ & 1.38 & 1.35 & 1.29 & 1.23 & 1.46 & 1.45 \\
\hline 34.56 & Isoeugenol & 164 & $\mathrm{C} 10 \mathrm{H} 12 \mathrm{O} 2$ & 5.19 & 4.56 & 4.30 & 4.23 & 3.79 & 2.43 \\
\hline 35.07 & Dihydroeugenol & 166 & $\mathrm{C} 10 \mathrm{H} 14 \mathrm{O} 2$ & -- & 0.69 & -- & -- & -- & 0.55 \\
\hline 36.15 & Acetovanillone & 166 & $\mathrm{C} 9 \mathrm{H} 10 \mathrm{O} 3$ & -- & 0.44 & -- & -- & 0.62 & 0.54 \\
\hline 37.91 & $\begin{array}{l}\text { (4-Hydroxy-3-methoxyphenyl)-2- } \\
\text { propanone }\end{array}$ & 180 & $\mathrm{C} 10 \mathrm{H} 12 \mathrm{O} 3$ & -- & -- & 1.03 & 1.39 & -- & -- \\
\hline 39.21 & 2-Tert-butyl-4-hydroxyanisole & 180 & $\mathrm{C} 11 \mathrm{H} 16 \mathrm{O} 2$ & 0.77 & 0.57 & 1.29 & 1.18 & 0.66 & -- \\
\hline
\end{tabular}




\begin{tabular}{|c|c|c|c|c|c|c|c|c|c|}
\hline 44.25 & 4-Allyl-2,6-dimethoxyphenol & 194 & $\mathrm{C} 11 \mathrm{H} 14 \mathrm{O} 3$ & 0.79 & 0.91 & 0.73 & 0.67 & 0.64 & 0.58 \\
\hline \multirow[t]{2}{*}{45.44} & Coniferyl aldehydel & 178 & $\mathrm{C} 10 \mathrm{H} 10 \mathrm{O} 3$ & 1.55 & 0.63 & 1.03 & 0.87 & 1.19 & -- \\
\hline & Anhydrosugars & & & 8.35 & 16.14 & 0.92 & 0.80 & 15.81 & 13.67 \\
\hline 14.1 & $\begin{array}{l}\text { 4-Hydroxy-5,6-dihydro-(2H)- } \\
\text { pyran-2-one }\end{array}$ & 114 & $\mathrm{C} 5 \mathrm{H} 6 \mathrm{O} 3$ & 2.12 & 7.16 & -- & -- & 1.27 & 2.96 \\
\hline 23.08 & $\begin{array}{l}\text { 1-Hydroxy-3,6- } \\
\text { dioxabicyclo[3.2.1]octan-2-one }\end{array}$ & 144 & C6H8O4 & 0.49 & 1.61 & -- & -- & 1.84 & 3.83 \\
\hline 36.9 & Levoglucosan & 162 & $\mathrm{C} 6 \mathrm{H} 10 \mathrm{O} 5$ & 5.74 & 7.37 & 0.92 & 0.80 & 12.70 & 6.89 \\
\hline
\end{tabular}

